\title{
The interrelationship of bone and cardiovascular remodeling biomarkers and clinical peculiarities of coronary artery disease in postmenopausal women
}

\author{
Natalia Serhiivna Mykhailovska ${ }^{1}$, Iryna Olehivna Stetsiuk ${ }^{1}$, Tamila Olehivna Kulynych ${ }^{1}$, \\ Svitlana Vasylivna Gorbachova², Igor Valentynovych Zhulkevych ${ }^{3}$ \\ ${ }^{1}$ Department of General Practice - Family Medicine and Internal Diseases, Zaporizhzhia State Medical University, Zaporizhzhia, Ukraine \\ 2Department of Clinical Laboratory Diagnostics, Zaporizhzhia State Medical University, Zaporizhzhia, Ukraine \\ ${ }^{3}$ Department of Oncology, Radiodiagnosis, Radiotherapy and Radiation Medicine, I. Horbachevsky Ternopil National Medical University, \\ Ternopil, Ukraine
}

\begin{abstract}
Objectives: To investigate the interrelationship between bone and cardiovascular remodeling biomarkers and some clinical, laboratory and instrumental parameters of coronary artery disease in postmenopausal women.

Material and methods: The double open cross-sectional monocentric clinical study in parallel groups involved 115 women in the postmenopausal period with coronary artery disease (CAD): stable exertional angina of functional class (FC) II-III (mean age $67.07 \pm 0.92$ years). Depending on the bone mineral density (BMD) state, all patients were divided into 3 groups: group $1(n=24)-$ patients with normal BMD (T-criterion more than $1 \mathrm{SD})$; group $2(n=34)$ - patients with osteopenia (T-criterion from -1 SD to $-2.5 \mathrm{SD}$ ); group $3(n=44)$ - patients with osteoporosis (T-criterion less than $-2.5 \mathrm{SD}$ ). The selected control group consisted of 12 relatively healthy women of the corresponding age. All patients underwent cardiovascular and bone remodeling biomarkers' assessment, lipid profile tests, daily monitoring of ECG by Holter, two-dimensional echocardiography and pulsed-wave Doppler. Intima-media complex (IMC) measurement, ultrasound densitometry, and the FRAX algorithm were performed.

Results: In women with coronary artery disease and postmenopausal osteoporosis (PMO), there was a significant increase in the level of bone osteoprotegerin (OPG), osteocalcin and vascular remodeling biomarkers (VEGF-A, homocysteine) in parallel with the progression of BMD disorders. The existence of a correlative dependence between osteoprotegerin level and the 10-year risk of fracture of the radial bone $(r=0.46 ; p<0.05)$ was discovered. A probable interrelationship between the OPG, osteocalcin, VEGF-A and homocysteine level and decrease of the T-criterion was observed. An interrelationship between the bone and cardiovascular remodeling biomarkers and atherogenic dyslipidemia, diastolic dysfunction of the left ventricle, dilatation of the left atrium, thickening of the intima-media complex, arrhythmic complications and vegetative imbalance development was proved $(p<0.05)$, which confirms their clinical and pathogenic role in patients with CAD and PMO. Conclusions: The results obtained prove the existing interrelationship of bone and cardiovascular remodeling biomarkers with the state of bone mineral density and clinical peculiarities of coronary artery disease in postmenopausal women.
\end{abstract}

Key words: coronary artery disease, postmenopausal osteoporosis, serum biomarkers, prognostic factors.

\footnotetext{
Address for correspondence:

Iryna Olehivna Stetsiuk, Department of General Practice, Zaporizhzhia State Medical University, 26 Maiakovskyi Ave, 69035 Zaporizhzhia Oblast, Ukraine, e-mail: iryna.zsmu@gmail.com

Submitted: 30.04.2020; Accepted: 4.06.2020
} 


\section{Introduction}

In contemporary internal diseases clinical picture comorbidity - the phenomenon when one patient has multiple internal organ diseases interrelated with a single pathogenic mechanism - is extremely common [1-4]. This problem is of particular social and medical importance in those cases when combined nosologies provide a significant impact on the quality and life expectancy of patients [1, 2, 5-8]. A large proportion of comorbid conditions is made up of pathologies of the circulatory system and musculoskeletal system, particularly the combination of coronary artery disease and osteoporosis [9].

Currently, there are only a few studies dedicated to the interrelation of coronary artery disease with bone mineral density (BMD) disorders. Existing literature provides information on only some aspects of this problem, firstly, regarding the prevalence and severity of vascular atherosclerosis and aortic calcification in patients with PMO $[10,11]$.

However, there is scientific evidence that coronary artery disease and osteoporosis have common progression mechanisms involving the cascade of proinflammatory cytokines, OPG, osteocalcin, endothelial dysfunction, estrogens, calcium deficiency, etc. [12, 13].

Therefore, the combination of coronary artery disease and osteoporosis requires further research of pathogenetic interrelationships in order to define criteria for early diagnosis and develop specific tactics for patient management [14]. Today, it is promising for this purpose to determine the level of biomarkers, reflecting the state of bone and cardiac remodeling, in particular the level of OPG, osteocalcin, homocysteine and vasculoendothelial growth factor (VEGF) [14].

However, the clinical and pathogenetic role of biomarkers of cardiovascular and bone remodeling taken with coronary artery disease requires further scientific research.

The aim was to investigate the interrelationship between bone and cardiovascular remodeling biomarkers and some clinical, laboratory and instrumental parameters of coronary artery disease in postmenopausal women.

\section{Material and methods}

The double open cross-sectional monocentric clinical study in parallel groups involved 115 women in the postmenopausal period with CAD: stable exertional angina of functional class (FC) II-III (mean age 67.07 \pm 0.92 years). Depending on the BMD state, all patients were divided into 3 groups: group $1(n=24)$ - patients with normal BMD (T-criterion more than $1 \mathrm{SD})$; group $2(n=$ 34 ) - patients with osteopenia (T-criterion from -1 SD to $-2.5 \mathrm{SD})$; group $3(n=44)$ - with osteoporosis (T-criterion less than -2.5 SD). The selected control group consisted of 12 relatively healthy women of the corresponding age.

In the following study the principles of bioethics were respected: the main provisions of the European Convention on Human Rights and Biomedicine (from 04.04.1997), GCP (1996), Helsinki Declaration of the World Medical Association on the Ethical Principles of Human Medical Scientific Research (1964-2000) and the Ministry of Health of Ukraine Order No. 281 dated 01.11.2000.

Criteria for the patient to be included in the study were the following: presence of verified (documented) stable exertional angina of FC II-III the duration of the postmenopausal period in women of more than 5 years, informed consent of the patient.

The exclusion criteria: chronic heart failure of NYHA FC III-IV; acute myocardial infarction or unstable angina; valvular heart defects; acute infectious diseases; severe somatic diseases during exacerbation and decompensation; endocrine pathology (hypogonadism, diabetes mellitus, hyperparathyroidism, thyrotoxicosis, acromegaly, hyperprolactinemia, hypercorticism) and diseases that induce the development of secondary osteoporosis; cancer and systemic diseases; mental disorders; alcohol abuse, drug addiction.

Angina pectoris and its functional class were diagnosed according to a comprehensive analysis of complaints, physical examination data, laboratory and instrumental findings in accordance with generally accepted standards.

The postmenopausal period was stated in the case of natural menopause, defined as the permanent cessation of menstrual periods, determined retrospectively after a woman has experienced 12 months of amenorrhea without any other obvious pathological or physiological cause.

The bone mineral density state was assessed according to WHO criteria. For the screening assessment of the degree of BMD loss the ultrasound osteodensitometry on the Omnisense 7000 apparatus (Israel) with sensors for the phalanx of the finger, the radial and tibia bones was used. The study was performed on a non-dominant limb. This method was chosen due to its convenience and simplicity for screening diagnosis of BMD disorders.

The measurement results were evaluated by the following indicators:

- T-index - the number of standard deviations by which the value of the patient's ultrasound speed differed from a similar value of young people of the same gender,

- Z-index - the number of standard deviations by which the value of the patient's ultrasound speed differed from the similar value of people of the same age and gender. 
If osteoporosis was diagnosed according to the ultrasound osteodensitometry data, patients were referred for X-ray absorptiometry (the gold standard for diagnosis) in order to confirm the diagnosis. This method was performed on Medix DR (France) apparatus with the assessment of BMD of the proximal femur and lumbar spine.

The degree of BMD loss was evaluated according to the T-criterion (the value of standard deviations [SD] from the mean values of the "peak bone mass"): the decrease in BMD to -1 SD was regarded as normal, from -1 to -2.5 SD - osteopenia, more than -2.5 SD osteoporosis [15].

The FRAX BMI online calculator, developed for the Ukrainian population, was used to assess the 10-year risk of osteoporotic fractures [16]. It allows one to calculate the 10-year probability of a fracture of the femoral neck (FRAX hip) and other typical fractures (FRAX total) associated with osteoporosis (vertebrae, radial, humerus) at the age of 40 to 90 years without taking into account the data of DXA.

All patients underwent biochemical studies to determine total cholesterol (TC), triglycerides (TG), high - (HDL) and low-density (LDL) lipoprotein levels using a PLIVA-Lachema BIOLATEST reagent. Low-density lipoprotein levels were calculated using the Friedewald formula.

Holter ECG daily monitoring was performed using a Cardiosens K instrument (KhAl MEDICA, Ukraine). To determine the signs of electrical instability of the heart, we investigated the number of cardiac arrhythmias per day. While analyzing heart rate variability (HRV), the time-domain and frequency-domain methods recommended by the Committee of Experts of the North American Society of Stimulation and Electrophysiology, the European Society of Cardiologists, and the Ukrainian Association of Cardiologists were used.

Two-dimensional echocardiography and pulse-wave Doppler were performed using the Esaote MyLab $50 \mathrm{XVi-}$ sion ultrasound scanner under the generally accepted practice according to ASE/EAE recommendations (2011). Quantitative and qualitative characteristics of the state of the intima-media complex (IMC) of the common carotid arteries (CCA) were evaluated under ultrasound visualization in B-mode. An increase in the thickness of IMC greater than $0.9 \mathrm{~mm}$ was considered to be a marker of atherosclerotic vascular damage.

All patients underwent the study of osteocalcin (set of reagents by Immunodiagnostic Systems Limited, UK) osteoprotegerin (set of reagents by Bender MedSystems GmbH, Austria), VEGF-A (eBioscience, An Affymetrix Company, USA), homocysteine (set of reagents by Axis-Shield, UK) using the ELISA method on the en- zyme-linked immunosorbent analyzer "SIRIO S" (Italy) on the basis of the educational medical and laboratory center of the Zaporizhzhia State Medical University.

Statistical data processing was carried out using the method of variation statistics with the help of the software package "Statistica 13.0" (StatSoft Inc., no JPZ8041382130ARCN10-J), under the generally accepted practice. The pattern of the distribution of the studied variables was assessed using Shapiro-Wilk's criterion.

Quantitative characteristics were represented as $M \pm m$ (arithmetic mean \pm standard error of arithmetic mean) or Me (Q25; Q75) (median, 25 and 75 distribution quartiles) depending on the type of data distribution. Under the normal distribution, the validity of the differences was estimated using the Student's T-criterion; under the distribution different from normal, Mann-Whitney's non-parametric U-criterion was used.

Assessment of the interrelationship between pairs of independent indexes, expressed in a quantitative scale, was performed by calculating Pearson rank correlation coefficients (in normal distribution) or Spearman rank correlation coefficients (in a distribution different from normal). To quantify the interrelationship between the impact of a specific factor and the type of pathological changes, a relative risk (RR) analysis was performed, with a 95\% confidence interval (Cl) determined by constructing four-field tables.

When constructing the tables the following indicators were taken into account: presence/absence of atherogenic dyslipidemia, IMC thickening of the CCA over $0.9 \mathrm{~mm}$, dilation of the right and left ventricles, systolic (left ventricular ejection fraction less than 45\%) and diastolic dysfunction (less than 1 ratio), of the left ventricle (LV), cardiac arrhythmias, sympathetic-parasympathetic imbalance (LF/HF ratio greater than 2), and ischemic myocardial changes (ST segment depression episodes significant in depth and duration), decrease of the T-criterion (T-score less than $-1 \mathrm{SD})$. At relative risk $(\mathrm{RR})>1$, the probability of occurrence of an adverse outcome in the group with exposure to the risk factor is higher, and at $R R<1$ is lower than in individuals without exposure to the risk factor. Differences were considered statistically significant at $p<0.05$.

\section{Results}

As we stated in our previous publications, in women with CAD in the postmenopausal period, depending on the severity of BMD loss, a significant decrease in the $\mathrm{T}$ - and Z-criteria and an increase in the 10-year risk of development of osteoporosis fractures were observed [17].

We found an increase in the level of bone (OPG, osteocalcin) and vascular (VEGF-A, homocysteine) remod- 
eling biomarkers in women with CAD in parallel with the progression of BMD disorders compared to healthy individuals and to women with CAD and normal BMD indices (Figs. 1-4).

We also determined the relative risk of complicated course of CAD on the background of PMO, which made it possible to assess the interrelationship between the influence of a certain factor and the type of pathological changes. An integrated scheme of interrelation of

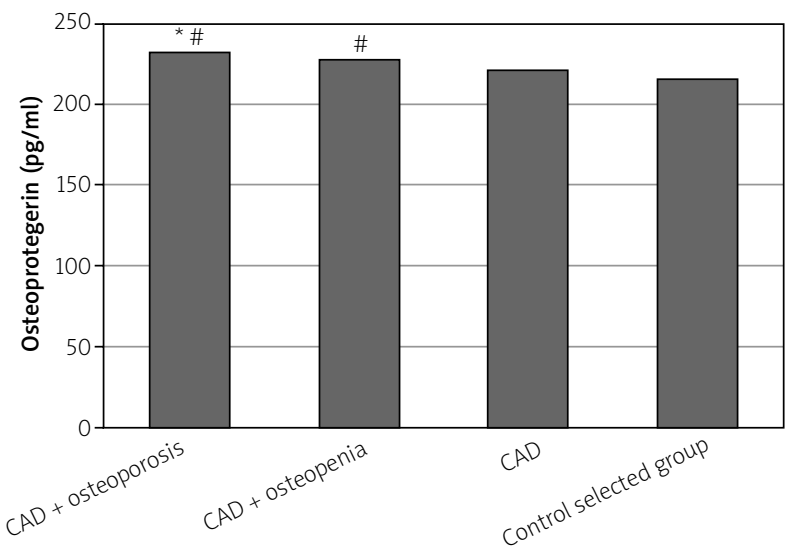

$C A D$ - coronary artery disease, $B M D$ - bone mineral density, \# probability of indexes difference compared to the control selected group $(p<0.05)$, * probability of indexes difference compared to the patients with $C A D$ and normal BMD state $(p<0.05)$.

Fig. 1. Level of osteoprotegerin in postmenopausal women with coronary artery disease, depending on the bone mineral density state.

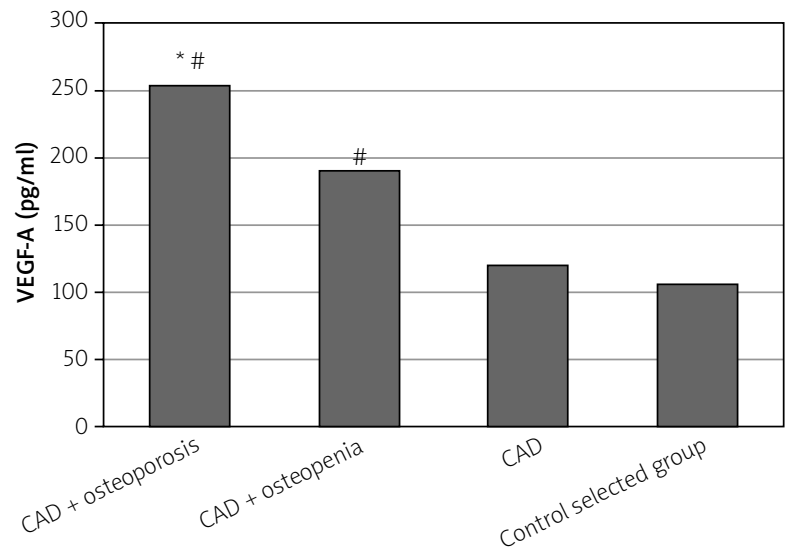

$C A D$ - coronary artery disease, $B M D$ - bone mineral density, \# probability of indexes difference compared to the control selected group $(p<0.05)$, * probability of indexes difference compared to the patients with $C A D$ and normal BMD state $(p<0.05)$.

Fig. 3. Level of vasculoendothelial growth factor A (VEGF-A) in postmenopausal women with coronary artery disease, depending on the bone mineral density state. the levels of biomarkers with the main indicators characterizing the clinical course of CAD on the background of BMD disorders is presented in Figure 5.

It was found that in women with CAD combined with BMD disorders an increase of osteocalcin level increased the relative risk of having sympathetic-parasympathetic imbalance (VNS imbalance) by 2.1 times ( $R R=2.143$; 95\% Cl: 1.013-4.532; $p<0.05)$, of atherogenic dyslipidemia by 1.5 times $(R R=1.540 ; 95 \% \mathrm{Cl}: 1.013-2.343$;

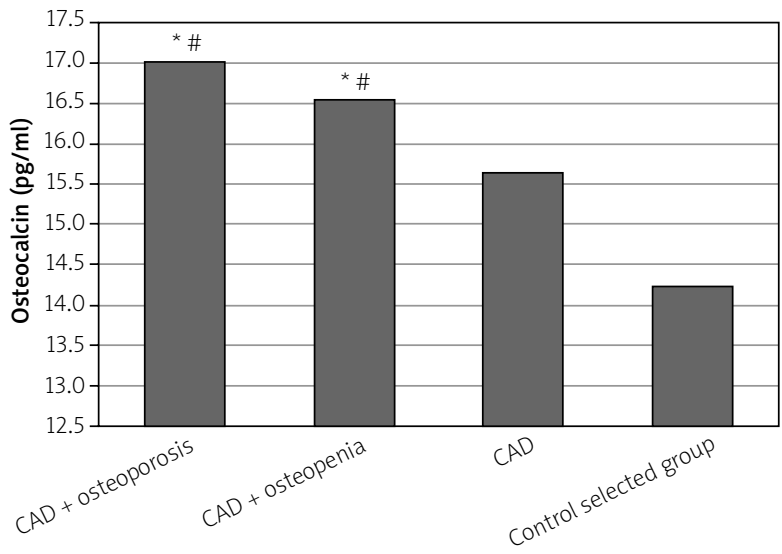

$C A D$ - coronary artery disease, $B M D$ - bone mineral density, \# probability of indexes difference compared to the control selected group $(p<0.05)$, * probability of indexes difference compared to the patients with CAD and normal BMD state $(p<0.05)$.

Fig. 2. Level of osteocalcin in postmenopausal women with coronary artery disease, depending on the bone mineral density state.

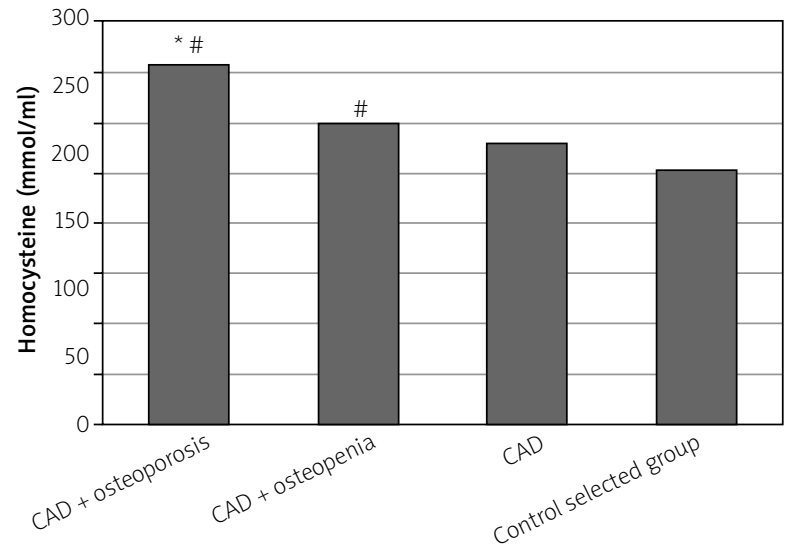

$C A D$ - coronary artery disease, $B M D$ - bone mineral density, \# probability of indexes difference compared to the control selected group $(p<0.05)$, * probability of indexes difference compared to the patients with CAD and normal BMD state $(p<0.05)$.

Fig. 4. Level of homocysteine in postmenopausal women with coronary artery disease, depending on the bone mineral density state. 


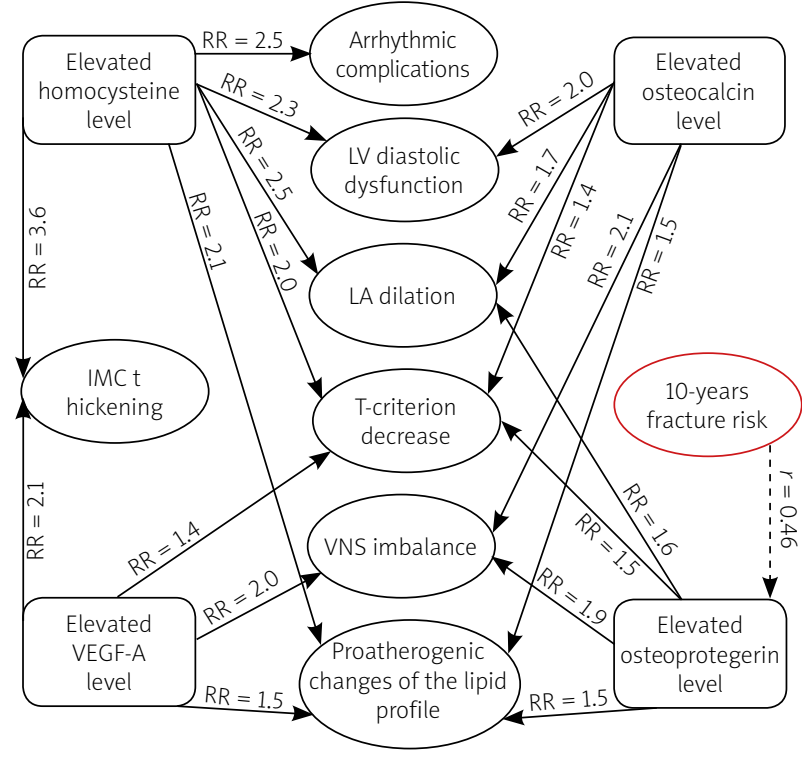

IMC - intima-media complex, $L A$ - left atrium, $L V$ - left ventricle, $R R$ relative risk, VEGF-A - vasculoendothelial growth factor-A, VNS - vegetative nervous system, $\longrightarrow R R$-relative risk, $--\rightarrow r$-correlative dependence.

Fig. 5. Interrelationship of level of biomarkers with clinical course of coronary artery disease on the background of bone mineral density disorders [acc. to 12,31 ].

$p<0.05)$, of diastolic dysfunction of the left ventricle (LV DD) by 2 times (RR = 2.027; 95\% Cl: 1.064-3.863; $p<0.05)$, of dilation of the left atrium (LA) by 1.7 times $(\mathrm{RR}=1.679 ; 95 \% \mathrm{Cl}: 1.026-2.749 ; p<0.05)$, of decrease of the T-criterion by 1.4 times $(\mathrm{RR}=1.445 ; 95 \% \mathrm{Cl}: 1.038$ 2.011; $p<0.05)$.

In women with CAD combined with BMD disorders an increase of OPG level increased the relative risk of having sympathetic-parasympathetic imbalance by 1.9 times (RR = 1.890; 95\% Cl: 1.040-3.435; $p<0.05)$, of atherogenic dyslipidemia by 1.5 times ( $R R=1.486 ; 95 \% \mathrm{Cl}$ : $1.045-$ 2.112; $p<0.05$ ), of dilation of the LA by 1.6 times ( $R R=$ 1.607; 95\% Cl: 1.004-2.574; $p<0.05$ ), of decrease of the T-criterion by 1.5 times ( $R R=1.455 ; 95 \% \mathrm{Cl}$ : 1.007-2.101; $p<0.05)$. Under the conduction of correlative analysis, the existence of a direct correlative dependence between osteoprotegerin level and the 10-year risk of fracture of the radial bone $(r=+0.46 ; p<0.01)$ was discovered.

In patients with CAD and BMD disorders an increase of VEGF-A level increased the relative risk of having an IMC thickened by 2.1 times (RR = 2.141; 95\% Cl: 1.040-4.408; $p<0.05)$, of sympatho-parasympathetic imbalance by 2 times ( $R R=2.040 ; 95 \% \mathrm{Cl}: 1.025-4.061 ; p<0.05)$, of atherogenic dyslipidemia by 1.5 times ( $R R=1.500 ; 95 \%$ $\mathrm{Cl}: 1.043-2.157 ; p<0.05)$, of decrease of the T-criterion by 1.4 times $(\mathrm{RR}=1.439 ; 95 \% \mathrm{Cl}: 1.004-2.063 ; p<0.05)$.
In patients with coronary artery disease and bone mineral density disorders an increase of homocysteine level increased the relative risk of having an IMC thickened by 3.6 times $(R R=3.556 ; 95 \% \mathrm{Cl}$ : 1.047-12.071; $p<0.05)$, of atherogenic dyslipidemia by 2.1 times ( $R R=$ 2.074; 95\% Cl: 1.052-4.090; $p<0.05)$, of LV diastolic dysfunction by 2.3 times $(R R=2.308 ; 95 \% \mathrm{Cl}$ : $1.126-4.731$; $p<0.05)$, of dilation of the LA by 2.5 times $(R R=2.528$; $95 \% \mathrm{Cl}: 1.052-6.072 ; p<0.05)$, of arrhythmic complications by 2.5 times ( $R R=2.566 ; 95 \% \mathrm{Cl}$ : 1.050-6.267; $p<0.05)$, of decrease of the T-criterion by 2 times $(R R=$ 1.980; $95 \% \mathrm{Cl}: 1.020-3.845 ; p<0.05)$.

\section{Discussion}

In groups of women with CAD and BMD disorders (osteopenia and osteoporosis) our study showed a significant increase in levels of the bone remodeling biomarkers osteoprotegerin and osteocalcin.

The results obtained can be explained by the fact that OPG is a key element in inhibiting the activation and differentiation of osteoclasts and is of great importance in bone resorption processes, and therefore its level is increased in case of violation of bone remodeling processes. The existence of a correlative interrelationship between the osteoprotegerin level and the 10-year risk of osteoporotic fractures proves the importance of the biomarker in the bone resorption processes in postmenopausal women.

According to Dzgoeva et al. [18], OPG is involved in the pathogenesis of vascular calcification, endothelial dysfunction and atherosclerosis. There is also evidence in the literature that OPG is a marker for the severity of the atherosclerotic process; its increased level leads to destabilization of the atherosclerotic plaque and the development of atherothrombosis [19-21]. Thus, the osteoprotegerin level may be considered as the link between arterial calcification and bone resorption, which underlie the clinical combination of vascular diseases and osteoporosis.

According to many authors, the increase in serum concentration of osteocalcin reflects the metabolic activity of osteoblasts of bone tissue, and also indicates its role in the regulation of the process of resorption, because under high levels of osteocalcin, bone resorption processes are also enhanced [22]. Therefore, it is both a general indicator of the level of bone metabolism and a possible prognostic indicator of the progression of osteoporosis. Also, many studies have revealed its involvement in the processes of vascular calcification, which occur with the progression of the atherosclerotic process [22, 23].

In our study, we observed an increase in the level of VEGF-A, which is a marker of neoangiogenesis synthe- 
sized by endothelial cells, vascular smooth muscle cells and macrophages in response to tissue ischemia [24].

According to the literature data, it is a powerful mitogen that promotes endothelial cell survival, increases vascular wall permeability, and regulates and accelerates the development of collateral circulation of ischemic myocardium in cardiovascular pathology [25].

However, overexpression of VEGF-A may also result in malfunction of the normal molecular mechanisms of ossification development and activation of osteoclastogenesis with the subsequent stimulation of osteoclast-mediated bone resorption [17].

The obtained data on the increase in homocysteine, which was the highest in women with CAD and PMO, can be explained by the formation of endothelium-dependent vasodilation and oxidative stress, the development of lipid peroxidation due to the increased synthesis of superoxide dismutase, thrombogenesis activation, and coagulation $[26,27]$.

In addition, in recent years hyperhomocysteinemia has been considered as a new marker for the risk of osteoporosis and osteoporotic fractures, because hyperhomocysteinemia contributes to the reduction of bone blood supply and is able to influence its biomechanical properties [27, 28].

In recent years, it has been established that hyperhomocysteinemia also leads to the activation of osteoclastogenesis and the enhancement of bone resorption processes, which in aggregate with oxidative and proatherogenic damage of peripheral vessels and impaired vascular production of nitric oxide allows it to be considered as a common link of pathogenesis between CAD and PMO $[25,28]$.

The data obtained regarding the increase of the risk of developing a sympatho-parasympathetic imbalance in patients with CAD with BMD disorders confirm the opinion of Elefteriou [29] that postmenopausal women are characterized by an increase in the tone of the sympathetic division of the autonomic nervous system, which not only worsens the course of cardiovascular pathology, but also leads to the development of osteoporosis due to the influence on $\beta 2$-adrenoreceptors on the surface of osteoblasts [13, 30-32].

The involvement of serum lipoproteins not only in the atherosclerotic process, but also in the processes of development and differentiation of bone cells (osteoblasts and osteoclasts), has been proven in many experimental studies [13, 32].

We revealed the increased risk of atherogenic dyslipidemia which can be explained by the fact that in osteoporosis, as in atherosclerosis, the activation of lipid peroxidation occurs, the accumulation of which in the subendothelial layer of the arteries leads to their calci- fication and suppresses bone mineralization processes [2]. In addition, oxidized LDLs can potentially stimulate osteoporosis through osteoclast-mediated bone resorption $[13,25]$.

The data obtained on the relationship between bone mineralization disorders and the development of diastolic dysfunction coincide with the results of Wang et al. [33]. Thus, in the Wang study, BMD loss was identified as an independent predictor of LV diastolic dysfunction, which can be explained by the effect of chronic inflammation with the hyperproduction of cytokines, growth factors, prostanoids, nitric oxide, and the development of oxidative stress in combination with the endothelial dysfunction.

Furthermore, in women with CAD and BMD disorders an increased risk of developing maladaptive remodeling of the heart and blood vessels was found, which confirms the results of the research by Tsarenok [34], who noted that the presence of osteoporosis in postmenopausal CAD patients is associated with an increase in the parameters of structural and geometric remodeling of the heart with predominance of the remodeling by the type of concentric LV hypertrophy.

The increased risk of arrhythmic complications in patients with hyperhomocysteinemia confirms the data obtained by Snezhitsky et al. [3], who demonstrated that homocysteine can regulate the activity of matrix metalloproteinases 2 and 9, which in turn can lead to structural and electrical myocardial remodeling with subsequent development of arrhythmic complications.

Thus, the obtained results confirm the existing scientific data on the presence of general pathogenetic bases of growth of bone and vascular remodeling biomarker levels, impaired osteogenesis and clinical features of coronary artery disease in postmenopausal women with CAD $[11,16,31]$.

\section{Conclusions}

In postmenopausal women with $C A D$ the presence of BMD disorders (osteopenia, osteoporosis) is accompanied by an increase in the level of bone and vascular remodeling biomarkers (OPG, osteocalcin, VEGF-A, homocysteine).

An increase of the level of OPG, osteocalcin, VEGF-A and homocysteine increases the relative risk of decrease of the T-criterion as well as atherogenic dyslipidemia, diastolic dysfunction of the LV, dilatation of the LA, arrhythmic complications, vegetative imbalance and thickening of the intima-media complex development, which confirms their interrelationship with the state of bone mineral density and clinical peculiarities of coronary artery disease in postmenopausal women. 
The existence of a correlative interrelationship between the osteoprotegerin level and the 10-year risk of osteoporotic fractures according to the FRAX data may indicate the role of the biomarker in the bone resorption processes in postmenopausal women.

\section{Acknowledgments}

The research was carried out within the scientific research work plan of the Department of General Practice and Family Medicine and Internal Diseases of the Zaporizhzhia State Medical University on the topic: "Clinical and pathogenetic, prognostic and treatment and diagnostic aspects of cardiovascular pathology with different comorbid states" (State registration number 0118U007138).

The authors declare no conflict of interest.

\section{References}

1. Zhuravlyova LV, Oliinyk MO. Features of bone metabolism in patients with osteoarthritis, type 2 diabetes mellitus and their combination. Pain Joints Spine 2018; 8: 85-92, DOI: 10.22141/2224-1507.8.2.2018.137189.

2. Kovalenko VM, Bortkevych OP. Comorbidity: definitions, possible directions of diagnosis and treatment. Ukrainian Rheumatology Journal 2019; 3: 33-44.

3. Snezhitsky VA, Yatskevich ES, Doroshenko EM, et al. Homocysteine as a prognostic marker of atrial remodeling and clinical picture in patients with paroxysmal and persistent forms of atrial fibrillation. Clin Med 2016; 94: 16-22, DOI: 10.18821/0023-2149-2016-94-1-16-22.

4. Smiyan S, Galaychuk I, Zhulkevych I, et al. Sjögren's syndrome and lymphadenopathy unraveling the diagnosis of Lyme disease. Reumatologia 2019; 57: 59-62, DOI: 10.5114/ reum.2019.83242.

5. Babynets LS, Maevska TG. Influence of concomitant chronic pancreatitis on prooxidant-antioxidant status and other parameters in osteoarthritis. Semejnaya Medicina 2016; 68: 75 78.

6. Mykhailovska NS, Oliynyk TV. Clinical and pathogenetic role of immunoinflammatory activation and endothelial dysfunction in patients with coronary heart disease associated with hypothyroidism based on the results of cognitive modeling. Zaporozhye Med J 2017; 19: 4-8, DOI: 10.14739/2310-1210. 2017.1.91585.

7. Mykhailovska NS, Miniailenko LE. Dynamics of structural, functional and vegetative changes of heart under the influence of combined therapy with the use of ursodeoxycholic acid and L-arginine in patients with coronary heart disease associated with nonalcoholic fatty liver disease. Patologia 2017; 2: $142-148$.

8. Mitchenko OI, Mamedov MN, Kolesnik TV, et al. Features of cardiovascular risk factors in women depending on menopause. Ukr Cardiol J 2015; 4: 96-102.
9. Nishkumay OI. Calcification of blood vessels, atherosclerosis and osteoporosis: is there a relationship? Heart and Blood Vessels 2016; 1: 107-112.

10. Ahmadi N, Mao SS, Hajsadeghi F, et al. The relation of low levels of bone mineral density with coronary artery calcium and mortality. Osteoporos Int 2018; 29: 1609-1616, DOI: 10.1007/ s00198-018-4524-7.

11. Mykhailovska NS, Stetsiuk IO. The interrelationship between the cardiovascular remodeling indicators and the state of bone mineral density in women with coronary artery disease. Patologia 2019; 1: 53-59.

12. Sagalovski S, Richter T. Vascular calcification and osteoporosis: from understanding common cellular and molecular mechanisms to search molecules as potential therapeutic targets. Ukr Cardiol J 2016; 1: 81-92.

13. Smiyan SI, Mitchenko OI, Kuzmina AP, et al. Diagnosis and monitoring of systemic connective tissue diseases: emphasis on laboratory-instrumental methods. Ukrainian Rheumatology Journal 2019; 3: 27-32.

14. Mattera M, Reginelli A, Bartollino S, et al. Imaging of metabolic bone disease. Acta Biomed 2018; 89: 197-207, DOI: 10.23750/ abm.v89i1-S.7023.

15. Povorozniuk VV, Grigorieva NV, Kanis JA, et al. Ukrainian version of FRAX: from creation to validation. Pain, joints, spine 2016; 3: 5-14, DOI: 10.22141/2224-1507.3.23.2016.85000.

16. Mykhailovska NS, Stetsiuk IO. The indicators of the bone tissue mineralization abnormalities in women with coronary artery disease in the post-menopausal period. Patologia 2018; 2: 136-141.

17. Galaychuk IY, Zhulkevych IV, Smiyan SI, et al. Borrelious lymphadenopathy in oncology practice (clinical observations). Oncology 2019; 21: 250-253.

18. Dzgoeva FU, Sopoev MY, Salamova EE, et al. Osteoprotegerin and RANKL: a role in the development of cardiovascular complications in patients with terminal stage of renal failure receiving hemodialysis. Nephrology (Saint-Petersburg) 2017; 21: 28-35, DOI: 10.24884/1561-6274-2017-21-5-25-33.

19. Polonskaya YaV, Kashtanova EV, Murashov IS, et al. Associations of osteocalcin, osteoprotegerin, and calcitonin with inflammation biomarkers in atherosclerotic plaques of coronary arteries. Bull Exp Biol Med 2017; 162: 726,-729, DOI: 10.1007/ s10517-017-3698-x.

20. Majerczyk M, Wajda J, Holecki M, et al. Osteoprotegerin as a marker of atherosclerosis and a prognostic factor in stroke. Postepy Hig Med Dos 2015; 69: 1505-1511, DOI: 10.5604/01.3001.0009.6620.

21. Demkova K, Kozarova M, Malachovska Z, et al. Osteoprotegerin concentration is associated with the presence and severity of peripheral arterial disease in type 2 diabetes mellitus. Vasa 2018; 47: 131-135, DOI: 10.1024/0301-1526/a000682.

22.Zhang X, Shen Y, Xu Y, et al. Association of serum osteocalcin levels with major adverse cardiovascular events: a 4.4-year retrospective cohort study. Clin Exp Pharmacol Physiol 2018; 45: 3-9, DOI: 10.1111/1440-1681.12853.

23. Tacey A, Qaradakhi T, Brennan-Speranza T, et al. Potential role for osteocalcin in the development of atherosclerosis and blood vessel disease. Nutrients 2018; 10: 1426, DOI: 10.3390/ nu10101426. 
24. Mohylnitska LA. Vasculoendothelial growth factor and endothelium-dependent dilation in patients with juvenile diabetes mellitus type 1 with microangiopathies and in young patients with obesity. Clinical Endocrinology and Endocrine Surgery 2015; 3: 23-29.

25. Huang H, Ma L, Kyrkanides S. Effects of vascular endothelia growth factor on osteoblasts and osteoclasts. AJO-DO 2016; 149: 366-373, DOI: 10.1016/j.ajodo.2015.09.021.

26. Korost YV. Hyperhomocysteinemia: a marker of vitamin B deficiency and an independent risk factor for developing cardiovascular disease. Liky Ukrayiny 2018; 1: 33-36.

27. Kutelmakh OI. Interconnection of vitamin D, homocysteine and dental diseases (literature review). Topic al Issues in Pharmaceutical and Medical Science and Practice 2019; 1: 104 112, DOI: 10.14739/2409-2932.2019.1.159166.

28. Babayeva NZ, Gasymov SE. The relationship between the concentration of homocysteine in the blood and changes in the vessels in patients with arterial hypertension. Meditsinskiye Novosti 2017; 1: 73-75.

29. Elefteriou F. Impact of the Autonomic Nervous System on the Skeleton. Physiol Rev 2018; 98: 1083-1112, DOI: 10.1152/ physrev.00014.2017.
30. Kryvenko VI, Borodavko OI. Oxidative stress features in patients with combined course of type 2 diabetes mellitus and osteoporosis residing in anthropogenic pressure conditions. Zaporozhye Medical Journal 2019; 2: 182-186, 10.14739/23101210.

31. Mykhailovska NS, Stetsiuk IO, Zelenina OO, et al. Research of the features of cardiovascular remodeling and bone tissue metabolism markers changes in women with coronary artery disease, associated with postmenopausal osteoporosis. Science Rise: Medical Science 2016; 8: 51-58.

32. Babinets LS, Halabitska IM. Chronic inflammatory process and bone tissue changes in patients with osteoarthritis and exocrine pancreatic insufficiency. Lek Obz (Med Horizon), 69, 2020; 1: 512-513.

33. Wang R, Li X, Zhang J, et al. Bone mineral density is associated with left ventricular diastolic function in women. Clin Cardiol 2016; 39: 709-714, DOI: 10.1002/clc.22592.

34. Tsarenok S. Structural and functional changes of myocardium in women with osteoporosis in combination with ischemic heart disease. The Clinician 2017; 11: 50-58, DOI: 10.17650/1818-8338-2017-11-3-4-50-58. 